\title{
Optimal Design for Minimizing Uncertainty in Dynamic Equilibrium Systems
}

\author{
H.T. Banks, Robert Baraldi, Kevin Flores \\ Department of Mathematics \\ Center for Research in Scientific Computation \\ North Carolina State University \\ Raleigh, $\mathrm{NC}$
}

March 7, 2015

\begin{abstract}
We further develop and utilize a previously described optimal design framework to investigate an oscillatory system of 16 states which simulates the circadian rhythm and intertwined positive and negative regulatory loops of Per, Cry, Bmal1, and Clock genes in mammals. We illustrate use of a subset selection methodology with experimental perturbations in order to increase and possibly maximize the amount of information gained from longitudinal data derived from such experiments. We demonstrate that optimizing experimental perturbations may substantially decrease uncertainty in estimating model parameters.
\end{abstract}

Key Words: Optimal experimental design, uncertainty analysis, sensitivity calculation, complex-step method, circadian rhythms. 


\section{Introduction}

Current efforts in modeling the host immune response to HIV infection and RNA3 recruitment in the Brome Mosiac Virus (BMV) replication cycle have elucidated the relationship between disturbances that drive biological systems away from equilibrium and information content in such perturbations $[2,1]$. For instance, the BMV model developed by Banks, et al. [4, 1] describes how experimental input manipulation can produce non-equilibrium system dynamics, which leads to a greater information content in collected data. The findings in [1] suggest that input manipulation is a powerful tool for reducing standard errors in parameter estimates. In the HIV model developed by Banks, et al. [2], anti-retroviral therapy (ART) drives viral load in patients toward an equilibrium level that is undetectable by ultra-sensitive assays. When ART is interrupted, often due to patient negligence, the HIV model converges to an equilibrium with high viral load. One of the results of [2] was that as the authors fit the HIV model to clinical patient data, the number of HIV model parameters that could be easily estimated with high statistical confidence increased with the number of treatment interruptions. Thus, non-equilibrium dynamics induced by ART perturbations increased the data information content as calculated through asymptotic standard errors for estimated model parameters.

In this paper, we look at the effects on parameter estimation that results from perturbing a dynamic system away from its oscillatory steady state. We hypothesize that disturbing an oscillatory system away from equilibrium yields better information for parameter estimation than an unaltered or undisturbed system, as evidenced by providing more statistical certainty in parameters for the model. To investigate this, we analyzed a mathematical model describing the regulation of genes involved in mammalian circadian rhythms. The expression of these genes can be inhibited through silencing their respective mRNAs. This permits the researcher to effectively negate the expression of a certain gene, thereby perturbing the system away from its periodic equilibrium. We employed an optimal experimental design theory framework described in [1], and further developed the algorithm to minimize parameter standard errors by choosing optimal times to perturb the model away from its dynamic equilibrium. Variations on the observation frequency and length of experiment are analyzed in the context of this algorithm, as well as the number of possible perturbations allowed. We report how optimization of experimentally controlled inputs, i.e., RNA inhibition (RNAi), for the circadian system can lead to a substantial decrease in standard errors for model parameters, thereby increasing the levels of certainty in parameters for the model.

\section{Mathematical model}

\subsection{Circadian rythms model}

The mathematical model of circadian rythms we used was previously developed in [9]; it is an oscillatory system of 16 state variables desribing a regulatory network of the Per, Cry, Bmal1, and Clock genes in mammals. Of the four parameter sets presented in [9], parameter set 1 of the model, described in Tables 1 and 2 of this paper and Table 2 of [9], models the protein and mRNA levels of the aforementioned genes in continuous darkness, which gives rise to the desired sustained circadian oscillations. The mechanism of circadian oscillation relies on the formation of an inactive complex between PER and CRY and the activators CLOCK and BMAL1 that enhance Per and Cry expression [9]. The occurence and period of these oscillations are generally most sensitive to parameters related to synthesis or degradation of Bmal1 mRNA or BMAL1 protein. The developers of this model concluded that sustained oscillations might arise from the sole negative autoregulation of Bmal1 expression. When the researchers integrated light-induced expression of the Per gene, the model accounted for rhythmic regulation of the oscillations by the light-dark cycle. Given the sustained oscillations of the model at steady state as well as the large number of system parameters, we found the model appropriate for assessing the impact of perturbing an oscillatory system away from the dynamic equilibrium on the uncertainty associated with estimating model parameters. The equations have been grouped by mRNAs, the phosphorylated and non-phosphorylated PER-CRY complex in the cytosol and nucleus, the phosphorylated and non-phosphorylated protein BMAL1 in the cytosol and nucleus, and the complex between PER-CRY and CLOCK-BMAL1 in the nucleus. The model, as developed in [9], is as follows:

(a) mRNAs of Per, Cry, and Bmal1: 


$$
\begin{aligned}
& \frac{d M_{P}}{d t}=v_{s P} \frac{B_{N}^{n}}{K_{A P}^{n}+B_{N}^{n}}-v_{m P} \frac{M_{P}}{K_{m P}+M_{P}}-k_{d m p} M_{P}, \\
& \frac{d M_{C}}{d t}=v_{s C} \frac{B_{N}^{n}}{K_{A C}^{n}+B_{N}^{n}}-v_{m C} \frac{M_{C}}{K_{m C}+M_{C}}-k_{d m c} M_{C}, \\
& \frac{d M_{B}}{d t}=v_{s B} \frac{K_{I B}^{m}}{K_{I B}^{m}+B_{N}^{m}}-v_{m B} \frac{M_{B}}{K_{m B}+M_{B}}-k_{d m b} M_{B} .
\end{aligned}
$$

(b) Phosphorylated and non-phosphorylated proteins PER and CRY in the cytosol:

$$
\begin{gathered}
\frac{d P_{C}}{d t}=k_{s P} M_{P}-V_{1 P} \frac{P_{C}}{K_{P}+P_{C}}+V_{2 P} \frac{P_{C P}}{K_{d p}+P_{C P}}+k_{4} P C_{C}-k_{3} P_{C} C_{C}-k_{d n} P_{C}, \\
\frac{d C_{C}}{d t}=k_{s C} M_{C}-V_{1 C} \frac{C_{C}}{K_{P}+C_{C}}+V_{2 C} \frac{C_{C P}}{K_{d p}+C_{C P}}+k_{4} P C_{C}-k_{3} P_{C} C_{C}-k_{d c} C_{C}, \\
\frac{d P_{C P}}{d t}=V_{1 P} \frac{P_{C}}{K_{P}+P_{C}}-V_{2 P} \frac{P_{C P}}{K_{d p}+P_{C P}}-v_{d P C} \frac{P_{C P}}{K_{d}+P_{C P}}-k_{d n} P_{C P}, \\
\frac{d C_{C P}}{d t}=V_{1 C} \frac{C_{C}}{K_{P}+C_{C}}-V_{2 C} \frac{C_{C P}}{K_{d p}+C_{C P}}-v_{d C C} \frac{C_{C P}}{K_{d}+C_{C P}}-k_{d n} C_{C P} .
\end{gathered}
$$

(c) Phosphorylated and non-phosphorylated PER-CRY complex in the cytosol and nucleus:

$$
\begin{aligned}
\frac{d P C_{C}}{d t}= & -V_{1 P C} \frac{P C_{C}}{K_{P}+P C_{C}}+V_{2 P C} \frac{P C_{C P}}{K_{d p}+P C_{C P}}-k_{4} P C_{C}+k_{3} P_{C} C_{C}+k_{2} P C_{N}-k_{1} P C_{C}-k_{d n} P C_{C} \\
\frac{d P C_{N}}{d t}= & -V_{3 P C} \frac{P C_{N}}{K_{P}+P C_{N}}+V_{4 P C} \frac{P C_{N P}}{K_{d p}+P C_{N P}}-k_{2} P C_{N}+k_{1} P C_{C}-k_{7} B_{N} P C_{N}+k_{8} I_{N}-k_{d n} P C_{N} \\
& \frac{d P C_{C} P}{d t}=V_{1 P C} \frac{P C_{C}}{K_{P}+P C_{C}}-V_{2 P C} \frac{P C_{C P}}{K_{d p}+P C_{C P}}+-v_{d P C C} \frac{P C_{C P}}{K_{D}+P C_{C P}}-k_{d n} P C_{C P} \\
& \frac{d P C_{N} P}{d t}=V_{3 P C} \frac{P C_{V}}{K_{P}+P C_{V}}-V_{4 P C} \frac{P C_{N P}}{K_{d p}+P C_{N P}}+-v_{d P N C} \frac{P C_{N P}}{K_{D}+P C_{N P}}-k_{d n} P C_{N P} .
\end{aligned}
$$

(d) Phosphorylated and non-phosphorylated protein BMAL1 in the cytosol and nucleus:

$$
\frac{d B_{C}}{d t}=k_{s B} M_{B}-V_{1 B} \frac{B_{C}}{K_{P}+B_{C}}+V_{2 B} \frac{B_{C} P}{K_{d p}+B_{C P}}-k_{5} B_{C}+k_{6} B_{N}-k_{d n} B_{C}
$$




$$
\begin{gathered}
\frac{d B_{C P}}{d t}=V_{1 B} \frac{B_{C}}{K_{P}+B_{C}}-V_{2 B} \frac{B_{C} P}{K_{d p}+B_{C P}}-v_{d B C} \frac{B_{C P}}{K_{d}+B_{C P}}-k_{d n} B_{C P}, \\
\frac{d B_{N}}{d t}=-V_{3 B} \frac{B_{N}}{K_{P}+B_{N}}+V_{4 B} \frac{B_{N P}}{K_{d p}+B_{N P}}+k_{5} B_{C}-k_{6} B_{N}-k_{7} B_{N} P C_{N}+k_{8} I_{N}-k_{d n} B_{N}, \\
\frac{d B_{N P}}{d t}=V_{3 B} \frac{B_{N}}{K_{P}+B_{N}}-V_{4 B} \frac{B_{N P}}{K_{d p}+B_{N P}}-v_{d B N} \frac{B_{N P}}{K_{d}+B_{N P}}-k_{d n} B_{N P} .
\end{gathered}
$$

(e) Inactive complex between PER-CRY and CLOCK-BMAL1 in the nucleus:

$$
\frac{d I_{N}}{d t}=-k_{8} I_{N}+k_{7} B_{N} P C_{N}-v_{d I N} \frac{I_{N}}{K_{d}+I_{N}}-k_{d n} I_{N} .
$$

Definitions of all the parameters used in these equations are in Tables 1 and 2. In Equations (1)-(16), concentrations are defined with respect to total cell volume. The initial conditions for this system are $I C=$ $\{0,0,8.7,0,0,0,0,0,0,0,0,0,0,0,0,0\}$; all are zero except the amount of Bmal1 mRNA. The concentration of every protein species (single or complex) is denoted by a subscript $C, N, C P$, or $N P$ for the cytosolic, nuclear, cytosolic phsophorylated or nuclear phosphorylated form, respectively.

Due to the substantial number of parameters present in the model and the combinatorial nature of the parameter subset selection method detailed in Section 4, we first had to reduce the number of parameters that were assumed to be estimated. In [9], the authors state that many parameter values remain to be determined experimentally, and that the oscillations created were obtained for a semi-arbitrary choice of parameter values in a physiologically realistic range. Given the somewhat arbitrariness of the parameter values and the implied difficulty of experimental estimation, we selected parameters based on mathematical type and biological significance. We also tried to select parameters that would represent a varying degree of sensitivity, and gathered this sensitivity information based on the values in Table 2 of [9]. Parameters $k_{1}$ through $k_{8}$ are all rate constants for the protein complexes described in the model, and act as decay or growth rates. Parameters $K_{A P}, K_{A C}$, and $K_{I B}$ describe the activation or inhibition of mRNA's by nuclear BMAL1, and appear in the model as Michaelis-Menten constants. Parameters $K_{d}, K_{d p}, K_{P}, K_{m B}, K_{m C}$ and $K_{m P}$ are all MichaelisMenten constants for proteins and mRNA's, and describe either degradation or phosphorylation processes. The parameter set that we apply the subset selection algorithm, developed in [3] and employed in [2, 1], is $P=\left\{k_{1}, k_{2}, k_{3}, k_{4}, k_{5}, k_{6}, k_{7}, k_{8}, K_{A P}, K_{A C}, K_{I B}, k_{d n}, K_{d}, K_{d p}, K_{P}, K_{m B}, K_{m C}, K_{m P}\right\}$, which amounts to 18 out of the 55 total model parameters. According to [9], the parameter set $P$ yields a diverse range of sensitivities, letting us observe the effects of the optimization algorithm on parameters with a variable amount of influence on the model solution. Reducing the number of parameters that will undergo the subset selection algorithm will also decrease the computational time to reasonable levels. The goal of using the circadian rythm model as an example is to demonstrate that perturbing an system with a dynamic equilibria can yield a data set with better information for parameter estimation than an unaltered or undisturbed system, thereby increasing statistical certainty in parameters estimated for the model. To create perturbations in the dynamic equilibria, we first developed a model for a experimental perturbation using mRNA inhibition (RNAi) based on biological principles. 


\begin{tabular}{|c|c|c|}
\hline Parameter & Definition & Values \\
\hline$k_{1}\left(h^{-1}\right)$ & Rate constant for entry of the PER-CRY complex into the nucleus & 0.4 \\
\hline$k_{2}\left(h^{-1}\right)$ & Rate constant for exit of the PER-CRY complex from the nucleus & 0.2 \\
\hline$k_{3}\left(n M^{-1} h^{-1}\right)$ & Rate constant for the formation of the PER-CRY complex & 0.4 \\
\hline$k_{4}\left(h^{-1}\right)$ & Rate constant for dissociation of the PER-CRY complex & 0.2 \\
\hline$k_{5}\left(h^{-1}\right)$ & Rate constant for entry of the BMAL1 protein into the nucleus & 0.4 \\
\hline$k_{6}\left(h^{-1}\right)$ & Rate constant for exit of the BMAL1 protein from the nucleus & 0.2 \\
\hline$k_{7}\left(n M^{-1} h^{-1}\right)$ & Rate constant for the formation of the inactive PER-CRY-CLOCK-BMAL1 complex & 0.5 \\
\hline$k_{8}\left(h^{-1}\right)$ & Rate constant for the dissociation of the PER-CRY-CLOCK-BMAL1 complex & 0.1 \\
\hline$K_{A P}(n M)$ & Activation constant for enhancement of Per expression by nuclear BMAL1 & 0.7 \\
\hline$K_{A C}(n M)$ & Activation constant for enhancement of Cry expression by nuclear BMAL1 & 0.6 \\
\hline$K_{I B}(n M)$ & Inhibition constant for repression of Bmal1 expression by nuclear BMAL1 & 2.2 \\
\hline$k_{d m b}\left(h^{-1}\right)$ & Nonspecific degradation rate constant for Bmal1 mRNA & 0.01 \\
\hline$k_{d m c}\left(h^{-1}\right)$ & Nonspecific degradation rate constant for Cry mRNA & 0.01 \\
\hline$k_{d m p}\left(h^{-1}\right)$ & Nonspecific degradation rate constant for Per mRNA & 0.01 \\
\hline$k_{d n c}\left(h^{-1}\right)$ & Nonspecific degradation rate constant for cytosolic non-phosphorylated CRY & 0.12 \\
\hline$k_{d n}\left(h^{-1}\right)$ & Nonspecific degradation rate for other protein species & 0.01 \\
\hline$K_{d}(n M)$ & Michaelis constant for protein degradation & 0.3 \\
\hline$K_{d p}(n M)$ & Michaelis constant for protein dephosphorylation & 0.1 \\
\hline$K_{P}(n M)$ & Michaelis constant for protein phosphorylation & 0.1 \\
\hline$K_{m B}(n M)$ & Michaelis constant for degradation of Bmal1 mRNA & 0.4 \\
\hline$K_{m C}(n M)$ & Michaelis constant for degradation of $C r y$ mRNA & 0.4 \\
\hline$K_{m P}(n M)$ & Michaelis constant for degradation of Per mRNA & 0.31 \\
\hline$k_{\text {stot }}\left(h^{-1}\right)$ & Rate constant for protein synthesis & 1.0 \\
\hline$k_{s B}\left(h^{-1}\right)$ & Rate constant for synthesis of BMAL1 & $0.12 k_{\text {stot }}$ \\
\hline$k_{s C}\left(h^{-1}\right)$ & Rate constant for synthesis of CRY & $1.6 k_{\text {stot }}$ \\
\hline$k_{s P}\left(h^{-1}\right)$ & Rate constant for synthesis of PER & $0.6 k_{\text {stot }}$ \\
\hline$n$ & Degree of cooperativity of activation of Per and Cry expression by BMAL1 & 4 \\
\hline$m$ & Degree of cooperativity of repression of Bmal1 expression by BMAL1 & 2 \\
\hline
\end{tabular}

Table 1: Parameters for the circadian rhythm model. 


\begin{tabular}{|c|c|c|}
\hline Parameter & Definition & Values \\
\hline$V_{p h o s}\left(n M h^{-1}\right)$ & Phosphorylation rate & 0.4 \\
$V_{1 B}\left(n M h^{-1}\right)$ & Maximum rate of cytosolic CRY phosphorylation & 0.5 \\
$V_{1 C}\left(n M h^{-1}\right)$ & Maximum rate of cytosolic PER phosphorylation & 0.6 \\
$V_{1 P}\left(n M h^{-1}\right)$ & Maximum rate of phosphorylation of cytosolic PER-CRY complex & $V_{p h o s}$ \\
$V_{1 P C}\left(n M h^{-1}\right)$ & Maximum rate of cytosolic BMAL1 dephosphorylation & $V_{p h o s}$ \\
$V_{2 B}\left(n M h^{-1}\right)$ & Maximum rate of cytosolic CRY dephosphorylation & 0.1 \\
$V_{2 C}\left(n M h^{-1}\right)$ & Maximum rate of cytosolic PER dephosphorylation & 0.1 \\
$V_{2 P}\left(n M h^{-1}\right)$ & Maximum rate of cytosolic PER-CRY complex dephosphorylation & 0.3 \\
$V_{2 P C}\left(n M h^{-1}\right)$ & Maximum rate of nuclear BMAL1 phosphorylation & 0.1 \\
$V_{3 B}\left(n M h^{-1}\right)$ & Maximum rate of phosphorylation of nuclear PER-CRY complex & 0.5 \\
$V_{3 P C}\left(n M h^{-1}\right)$ & Maximum rate of nuclear BMAL1 dephosphorylation & $V_{p h o s}$ \\
$V_{4 B}\left(n M h^{-1}\right)$ & Maximum rate of dephosphorylation of nuclear PER-CRY complex & 0.2 \\
$V_{4 P C}\left(n M h^{-1}\right)$ & Maximum rate of degradation of cytosolic phosphorylated BMAL1 & 0.1 \\
$v_{d B C}\left(n M h^{-1}\right)$ & Maximum rate of degradation of nuclear phosphorylated BMAL1 & 0.5 \\
$v_{d B N}\left(n M h^{-1}\right)$ & Maximum rate of degradation of cytosolic phosphorylated CRY & 0.6 \\
$v_{d C C}\left(n M h^{-1}\right)$ & Maximum rate of degradation of cytosolic phosphorylated PER & 0.7 \\
$v_{d I N}\left(n M h^{-1}\right)$ & Maximum rate of degradation of nuclear PER-CRY-CLOCK-BMAL1 complex & 0.8 \\
$v_{d P C}\left(n M h^{-1}\right)$ & Maximum rate of degradation of cytosolic phosphorylated PER-CRY complex & 0.7 \\
$v_{d P C C}\left(n M h^{-1}\right)$ & 0.7 \\
$v_{d P C N}\left(n M h^{-1}\right)$ & Maximum rate of degradation of nuclear phosphorylated PER-CRY complex & 0.7 \\
$v_{m B}\left(n M h^{-1}\right)$ & Maximum rate of Bmal1 mRNA degradation & 0.8 \\
$v_{m C}\left(n M h^{-1}\right)$ & Maximum rate of Cry mRNA degradation & 1.0 \\
$v_{m P}\left(n M h^{-1}\right)$ & Maximum rate of Per mRNA degradation & 1.1 \\
$v_{s T o t}\left(n M h^{-1}\right)$ & Maximum transcription rate & 1.0 \\
$v_{s B}\left(n M h^{-1}\right)$ & Maximum rate of Bmal1 mRNA synthesis & 1.1 \\
$v_{s C}\left(n M h^{-1}\right)$ & Maximum rate of Cry mRNA synthesis & $1.5 v_{s B}$ \\
$v_{s P}\left(n M h^{-1}\right)$ & Maximum rate of Per mRNA synthesis \\
\hline
\end{tabular}

Table 2: Parameters for the circadian rhythm model. 


\subsection{Model for system perturbations using RNAi}

We model the RNA inhibiter (RNAi) by introducing an additional state variable $r$ that represents the concentration of exogenously spawned small interfering RNAs (siRNA's) that can inhibit a target mRNA posttranscriptionally. The intracellular concentration of siRNA is modeled by the equation

$$
\frac{d r}{d t}=u(t) r_{o n}-r_{d} r
$$

The function $u(t)$ is a piecewise constant function with range in $\{0,1\}$ that represents whether the RNAi is on or off for each of $H$ time intervals $\left[t_{i-1}^{b}, t_{i}^{b}\right], i=1, \ldots, H$. The function $u(t)$ signifies whether the RNAi is active $(=$ 1 ) or inactive $(=0)$ in certain intervals of time. The parameter $r_{o n}$ represents the rate of siRNA influx into cells and $r_{d}$ is the siRNA exponential decay rate. The constant $r_{d}$ was taken to be 2 hours $^{-1}$ as described in [11]. Due to the lack of literature on the value of $r_{o n}$, we assume it is equal to 4 (concentration/hour), as this represents a seemingly reasonable rate of siRNA influx. It is assumed there is initially no silencer in the system $(r(0)=0)$. To simulate the effect of RNAi in the model, we had the RNAi influence the removal rate of the Per mRNA.

The description of RNAi was inserted into Equation (1) by creating a $\left(1+m_{r} r\right)$ term and multiplying it by the decay rate $k_{d m p} M_{P}$. Here, $m_{r}$ is the mass action interaction rate between $r$ and $M_{p}$. The modified equation for Per mRNA is now

$$
\frac{d M_{p}}{d t}=v_{s P} \frac{B_{N}^{n}}{K_{A P}^{n}+B_{N}^{n}}-v_{m P} \frac{M_{P}}{K_{m P}+M_{P}}-k_{d m p}\left(1+m_{r} r\right) M_{P},
$$

where $m_{r}$ is taken to be 10. It is important to note that while this may seem a high value, for simplification we did not want to affect more than one mRNA removal rate, and thus needed a slightly larger value to obtain a reasonable perturbation magnitude. Thus, we have implemented a state that will increase the rate at which Per mRNA is removed, and in combination with the function $u(t)$, effectively introduces perturbations into the system.

\section{Optimal experimental design theory}

\subsection{Uncertainty quantification with asymptotic theory}

We employed asymptotic theory in order to quantify parameter uncertainty within an optimal experimental design framework. Below, we formulate a Fisher Information Matrix, i.e., from asymptotic theory, for a given assumed level of observation noise and a function describing system perturbations, i.e., $u(t)$. In the model above (specifically Equation (18)), the function $u(t)$ is assumed to be known as it is controlled by the experimenter. With the addition of the RNAi, we can write the circadian model generally as

$$
\begin{array}{r}
\frac{d \vec{x}}{d t}(t)=\vec{g}(t, \vec{x}(t ; \hat{q}, u(t)), \hat{q}, u(t)), \quad t \in\left[t_{0}, t_{f}\right], \\
\vec{x}\left(t_{0}\right)=\vec{x}_{0}, \quad \vec{f}(t ; \hat{q}, u(t))=C \vec{x}(t ; \hat{q}, u(t))
\end{array}
$$

where $\vec{x}_{0}$ are given fixed initial conditions, $\vec{x}(t ; \hat{q}, u(t))$ is the vector of state variables of the system time point $t$ and parameter vector $\hat{q} \in \mathbb{R}^{p}$, with $p=18$ "estimated" parameters $P$ from the total set of 55 model parameters from Tables 1 and 2 . The initial time and final time are designated $t_{0}$ and $t_{f}$, respectively. Let $\mathcal{P}_{1}\left(\left[t_{0}, t_{f}\right]\right)$ denote the set of all bounded distributions on the interval $\left[t_{0}, t_{f}\right]$. The set of functions describing RNAi perturbations we use is the set of piecewise constant functions with a domain $\left[t_{0}, t_{f}\right]$ that is divided into $H$ intervals with variable length, and with a range in $\{0,1\}$. Specifically, let $S=\left\{s=\left(s_{0}, s_{1}, \ldots, s_{H}\right) \in \mathbb{R}^{H+1} \mid s_{i}<s_{i+1}\right.$ for $\left.i=0, \ldots, H-1\right\}$ and let $B=\mathbb{Z}_{2}^{H}$. Then the perturbation function is defined in a piecewise constant fashion as $u(t):=u(t ; b, s)=b_{i}$ for $t \in\left[s_{i-1}, s_{i}\right], i=1, \ldots, H$, where $b \in B$ and $s \in S$. Thus the set $U \simeq B \times S$ contains all of the admissible perturbation functions $u(t ; b, s)$. Let $\mathcal{P}_{2}(U)$ represent the set of all bounded distributions $\mathcal{P}_{2}(u)$ on $U$. Then the Generalized Fisher Information Matrix (GFIM) may be written

$$
\mathcal{F}\left(\mathcal{P}_{1}, \mathcal{P}_{2}, \hat{q}\right)=\int_{t_{0}}^{t_{f}} \int_{\mathbb{Z}_{2}^{H}} \nabla_{\hat{q}}^{T} \vec{f}(t ; \hat{q}, u(t))\left(V_{0}^{-1}(t)\right) \nabla_{\hat{q}} \vec{f}(t ; \hat{q}, u(t)) d \mathcal{P}_{2}(u) d \mathcal{P}_{1}(t) .
$$


Here $\hat{q}=\hat{q}_{0}$ is the assumed "true" parameter vector with the corresponding statistical error model (see Section 3.2 .2 of [6]), which we assume to be constant, given by

$$
\vec{Y}(t)=\vec{f}(t ; \hat{q}, u(t))+\widetilde{\mathcal{E}}(t),
$$

where $\vec{f}(t ; \hat{q}, u(t)) \in \mathbb{R}^{6}$ and the $\widetilde{\mathcal{E}}(t)$ are independent and identically distributed with zero mean and covariance matrix given by $V_{0}(t)$.

We consider the case of observations collected at discrete times where we choose a set of $n$ time points $\tau=\left\{t_{j}\right\}, j=1,2, \ldots, n$, and $t_{0}=t_{1}<t_{2}<\cdots<t_{n}=t_{f}$. The corresponding discrete $p \times p$ Fisher information matrix (FIM) for a perturbation function $u(t ; b, s)$ measured at discrete times $\tau$ is

$$
F(\tau, u, \hat{q})=\sum_{j=1}^{n} \nabla_{\hat{q}}^{T} \vec{f}\left(t_{j} ; \hat{q}, u\left(t_{j} ; b, s\right)\right)\left(V_{0}^{-1}\left(t_{j}\right)\right) \nabla_{\hat{q}} \vec{f}\left(t_{j} ; \hat{q}, u\left(t_{j} ; b, s\right)\right) .
$$

Here, $V_{0}$ is taken to be the $6 \times 6$ diagonal matrix $V_{0}=\operatorname{diag}\left(\sigma^{2}, \ldots, \sigma^{2}\right)$ and we assume for these calculations that the variance is equal to a constant value of $\sigma^{2}=1$ for all states. The values in the sensitivity matrices $\nabla_{\hat{q}} \vec{f}\left(t_{j} ; \hat{q}, u\left(t_{j}\right)\right)$ were calculated using the complex-step derivative method (see Section 3.2 ) and the parameters $\hat{q}$ supplied in $P$ from those in Tables 1 and 2 . Indeed, throughout we tacitly assume that these $\hat{q}$ resulted from a prior best fit parameters to data. The choice of optimal design criteria is given by the minimization of a functional $\mathcal{J}(\mathcal{F}): \mathbb{R}^{p \times p} \rightarrow \mathbb{R}^{+}$; we utilize SE-optimal design, introduced in [5]. For any component $q_{k}$ of the estimated parameter vector $\hat{q}$, the standard error $\left(S E_{k}\right)$ is computed by methods of asymptotic theory [7] that rely on the Fisher Information Matrix (Equation (22)).

\subsection{Complex-step derivative method for calculating sensitivities}

We briefly describe the complex-step derivative (see [10] for details) used to calculate an approximation for the sensitivities of the six species seen in Figure 2 of [9] with respect to model parameters. These species are: $M_{P}$, the mRNA of Per; $M_{C}$, the mRNA of Cry; $M_{B}$, the mRNA of Bmal1; $C_{T o t}$, the total amount of CRY; $P_{T o t}$, the total amount of PER, and $B_{T o t}$, the total amount of BMAL1. Essentially, the complex-step derivative is a finite-difference approximation calculated in the complex plane. Recall the forward-difference formula, wherein a common estimate for the first derivative of a scalar function $f(x)$ is

$$
f^{\prime}(x)=\frac{f(x+h)-f(x)}{h}+o(h),
$$

where $h$ is the finite-difference interval and $o(h)$ is the truncation error for the first-order approximation. Consider a function $f=u+i v$ of the complex variable $z=x+i y$. Paraphrasing [10], if $f$ is analytic in the complex plane then the Cauchy-Riemann equations apply and are given by

$$
\begin{gathered}
\frac{\partial u}{\partial x}=\frac{\partial v}{\partial y} \\
\frac{\partial u}{\partial y}=-\frac{\partial v}{\partial x} .
\end{gathered}
$$

Equations (24) and (25) give the relationship between the real and imaginary parts of the function. We can use the definition of a derivative in the right hand side of the first Cauchy-Riemann Equation (24) to write

$$
\frac{\partial u}{\partial x}=\lim _{h \rightarrow 0} \frac{v(x+i(y+h))-v(x+i y)}{h},
$$

where $h$ is a real number. Since the Equations (1)-(16) are real functions of real variables, $y=0, u(x)=f(x)$, and $v(x)=0$. Equation (26) can be rewritten as

$$
\frac{\partial f}{\partial x}=\lim _{h \rightarrow 0} \frac{\operatorname{Im}[f(x+i h)]}{h}
$$


and approximated by

$$
\frac{\partial f}{\partial x} \approx \frac{\operatorname{Im}[f(x+i h)]}{h} .
$$

The value of $h$ is taken to be very small, e.g., $h=10^{-50}$. This is the complex-step derivative approximation, and is not subject to subtractive cancellation errors since there is no difference operation [10]. This method was implemented for each of the 18 parameters selected and the 6 states described above. This method is used to calculate partial derivatives for the Fisher Information Matrix as defined in [7].

\subsection{Optimal experimental design algorithm}

The algorithm is initialized with an unoptimized or naive experimental design described by $u(t ; b, s)$, which depends on $s$, an ordered set of $H$ on/off intervals, and a binary vector $b \in \mathbb{Z}_{2}^{H}$. The naive experimental design corresponds to $b=\overrightarrow{0}$. For our results below, the vector $s$ was initialized (as $s^{*}$ ) using periodic spacing between the initial and final experiment times, $t_{0}$ and $t_{f}$, respectively. The experimental designs we consider here are for a fixed set of observation times $\tau$, which will consist of periodic observations (every 1 or 3 hours) between $t_{0}$ and $t_{f}$. We note that optimization of the observation times $\tau$ in addition to the experimental perturbation function $u(t ; b, s)$ may also be considered in future work and have been investigated elsewhere [1]. We also note that the algorithm we use here is different from that developed in [1], since we optimize rather than fix the time intervals defined by $s$. Calculating the optimal $u(t ; b, s)$ requires nonlinear optimization of $H-1$ time points, i.e., the boundary of the intervals defining the piecewise constant function, and $2^{H}$ possible input vectors for a total of $H-1+2^{H}$ dimensions. Instead of this computationally intensive procedure, we iteratively solve

$$
b^{*}=\underset{\left\{b \mid \mathcal{P}_{u} \in \mathcal{P}_{2}(U), s=s^{*}\right\}}{\operatorname{argmin}} \mathcal{J}(F(\tau, u(t ; b, s), \hat{q}))
$$

and

$$
s^{*}=\underset{\left\{b \mid \mathcal{P}_{u} \in \mathcal{P}_{2}(U), b=b^{*}\right\}}{\operatorname{argmin}} \mathcal{J}(F(\tau, u(t ; b, s), \hat{q}))
$$

by computing the sum of the normalized standard errors for the parameters in $P$ (SE-optimal design presented in [5]) for all $b \in \mathbb{Z}_{2}^{H}$, and by using using fminsearch in Matlab (The Mathworks, Natick, MA), respectively. The minimum sum is chosen, and the process iterates with the chosen $b$ and $s$ to define the new perturbation function $u(t ; b, s)$ for the experimental design. When the same $b$ and $s$ are selected twice in a row, it is assumed that these values define the optimal perturbation function. This optimal perturbation function is then used to produce uncertainty quantification plots using the parameter subset selection procedure.

\section{Parameter subset selection algorithm}

The parameter subset selection algorithm of [3, 8] was implemented in [2], and can be used to better understand how many parameters a data set may support for a given level of observation error. The algorithm selects a parameter subset based on minimizing the sum of normalized standard errors (selection score) for a certain number of estimated parameters $n_{p}$. If we have a set of size $p$ chosen from a set of parameters $\hat{q}$ (for this model $p=18$ ), and a number $n_{p} \leq p$, the subset selection algorithm finds a subset of parameters of size $n_{p}$ that minimizes a selection score as described in [3]. To implement this procedure one first needs a set of parameter estimates $\left\{\hat{q}_{1}, \ldots, \hat{q}_{p}\right\}$, with corresponding standard errors $\left\{S E_{1}, \ldots, S E_{p}\right\}$. One then introduces the coefficients of variation for $\hat{q}_{i}$

$$
\nu\left(\hat{q}_{i}\right)=\frac{S E_{i}}{\hat{q}_{i}}, \quad i=1, \ldots, n_{p},
$$

and calculates a selection score given by the Euclidean norm in $\mathcal{R}^{n_{p}}$ of $\nu(\hat{q})=\left(\nu\left(\hat{q}_{1}\right), \ldots, \nu\left(\hat{q}_{n_{p}}\right)\right)^{T}$, i.e.,

$$
\alpha(\hat{q})=|\nu(\hat{q})|
$$


as an uncertainty quantification for the estimates $\hat{q}$. The components of the vector $\nu(\hat{q})$ are the ratios of each standard error for a parameter to the corresponding nominal parameter value. These ratios are dimensionless numbers warranting comparison even when parameters have considerably different scales and units; thus, they are compared graphically on a logarithmic scale. Graphs such as Figure 2 below plot the $v\left(\hat{q}_{i}\right)$ for each parameter (y-axis) for $n_{p}=1, \ldots, 18$ (x-axis).

\section{Results}

We sought to determine whether optimizing system perturbations could improve confidence in parameters estimated for a system with a dynamic (i.e., periodic) equilibrium, using the circadian rhythms model as a test example. Below, we analyzed forward solutions and uncertainty quantification plots resulting from the parameter subset selection algorithm using a naive or optimal experimental design for several different choices of the final experiment time $\left(t_{f}\right)$, the observation frequency, and the number of possible perturbations $(H)$. The sensitivity matrix is evaluated at the discrete time points determined by the observation frequency. The forward solutions are presented in a similar style to those in Figure 2 of [9], where the total amount of Per, Cry, and Bmal1 mRNAs and PER, CRY, and BMAL1 proteins are presented. The parameter subset selection graphs are the plots of the normalized standard errors for each parameter in the set $P$. It is important to note that the subset selection algorithm does not re-estimate parameters when minimizing the selection score; the Fisher Information Matrix is calculated only once, from which respective rows and columns are removed to calculate standard errors. We note that re-estimation following the application of the subset selection algorithm was tested in [2] and proved to make only negligible differences in the results. We define a threshold at which parameter uncertainty can be taken as reasonable by setting an upper bound on the normalized standard error for each parameter in a given subset; this bound ensures that every $95 \%$ confidence interval is narrower than the parameter estimate itself. This value is

represented by the horizontal (red) line in the subset selection graphs with the value $\frac{1}{1.96}=0.5102$, as presented in $[2]$.

For reference, we plotted the forward solution of the model using the parameter set in Tables 1 and 2 without any optimized interruptions, i.e., using a naive experimental design (Figure 1). The forward solution is plotted for 0-72 hours as in [9] with an observation frequency of 3 hours. 

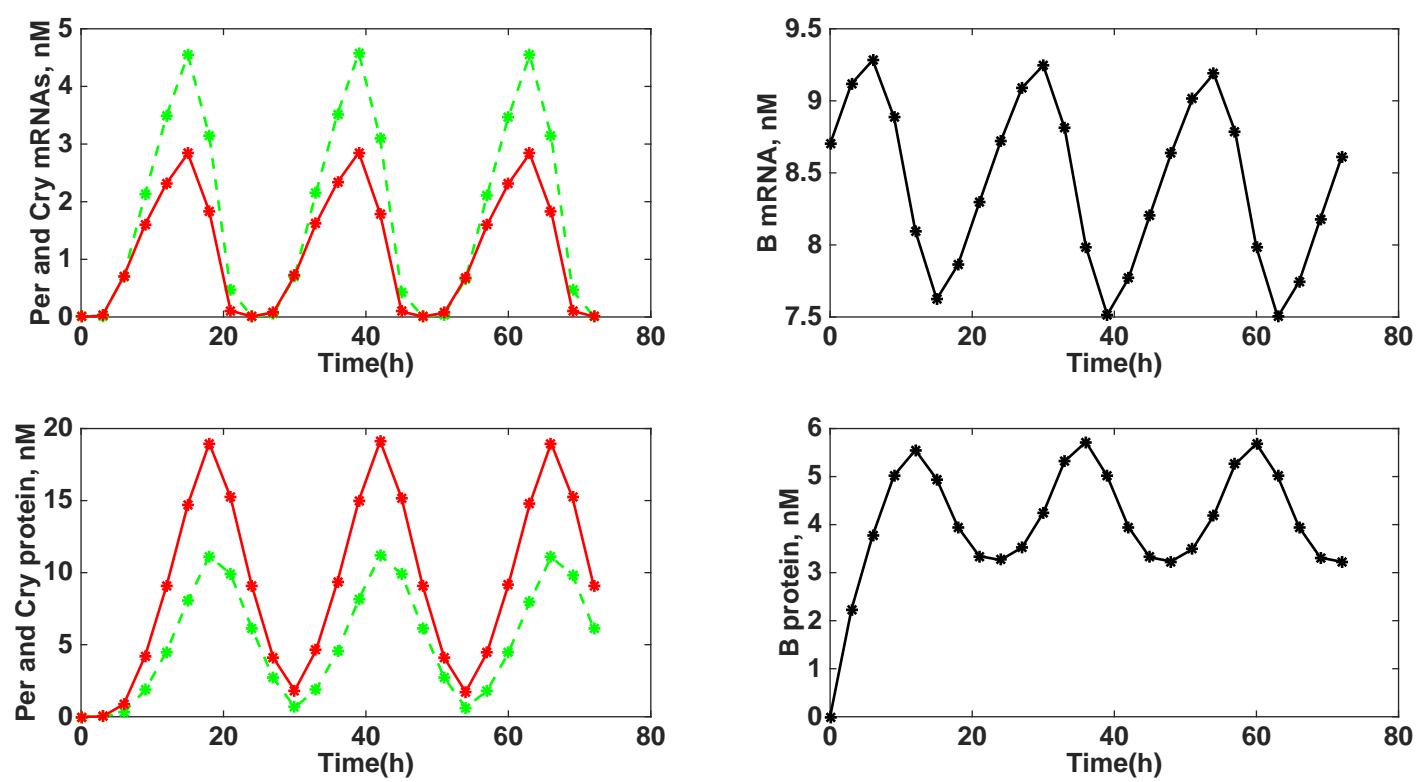

Figure 1: The forward solution of the circadian model for the Per, Cry, and Bmal1 (B) mRNAs and PER, CRY, and BMAL1 (B) proteins from 0-72 hours with the observation frequency equal to 3 hours. In the left figures, solid lines represent $\mathrm{Cr} y$ while dashed lines are Per. Forward solutions are calculated at discrete observation time points (every 3 hours) and plotted as $\left(^{*}\right)$, with connecting lines shown for clarity. 


\subsection{Optimal Experimental Design: $t_{f}=72 \mathrm{hrs}$, observation frequency $=3 \mathrm{hrs,}$ $H=3$}

We first investigated whether introducing RNAi perturbations, calculating using our optimal experimental design algorithm (Equations (29) - (30)), could have an affect on reducing parameter uncertainty. We implemented our optimization algorithm using an experimental time of 72 hours, an observation frequency of 3 hours, and 3 time intervals for possible RNAi activation/deactivation $(H=3)$. Using 3 possible perturbation intervals, we found that the optimal time mesh $s$ and perturbation vector $b$ corresponding to the function $u(t ; b, s)$ were equal to $\{0,16.8410,47.0857,72\}$ and $\{0,1,0\}$, respectively. Figure 2 (top) displays the naive experimental design results with comparison to the optimized experimental design results (bottom) with regard to parameter uncertainty as calculated using the subset selection algorithm. As stated previously, all of the parameter subset selection graphs were plotted on a logarithmic scale due to the substantial difference of the normalized standard errors (NSEs). Specifically, the NSEs for each parameter were calculated using a parameter subset selection algorithm and are plotted $\log _{10}$ scale for $n_{p}=1, \ldots, 18$ chosen parameters.

We found that an optimized experimental design produced improvement in parameter uncertainty. The sum of the NSEs at $n_{p}=18$ for the naive and optimal experimental design were 63.9709 and 43.2897 , respectively. We also observed an overall decrease in the NSEs for each of the parameters chosen by the subset selection algorithm at all values of $n_{p}=1, \ldots, 18$. For example, the NSE for $K_{m C}$ estimated at $n_{p}=18$ is less for the optimal experimental design (0.2144) than for the naive experimental design (0.4363), and similar results were found for the other parameters in the model at all values of $n_{p}$. Moreover, two parameters were below the $95 \%$ confidence interval threshold at $n_{p}=18$ vs. only 1 parameter for the naive experimental design (Figure 2). Specifically, only the NSE for $K_{m C}$ completely falls below the threshold set at .5102 for both naive and optimal experimental designs. However, the NSE for $k_{5}$ also falls below the threshold for the naive and experimental designs for all values of $n_{p}$. We also observed that RNAi perturbations could increase the number of parameters estimated with reasonable statistical confidence, i.e., the maximum number of parameters that lay below the uncertainty threshold were $n_{p}=8$ and $n_{p}=10$ for the naive and optimal experimental designs, respectively. 

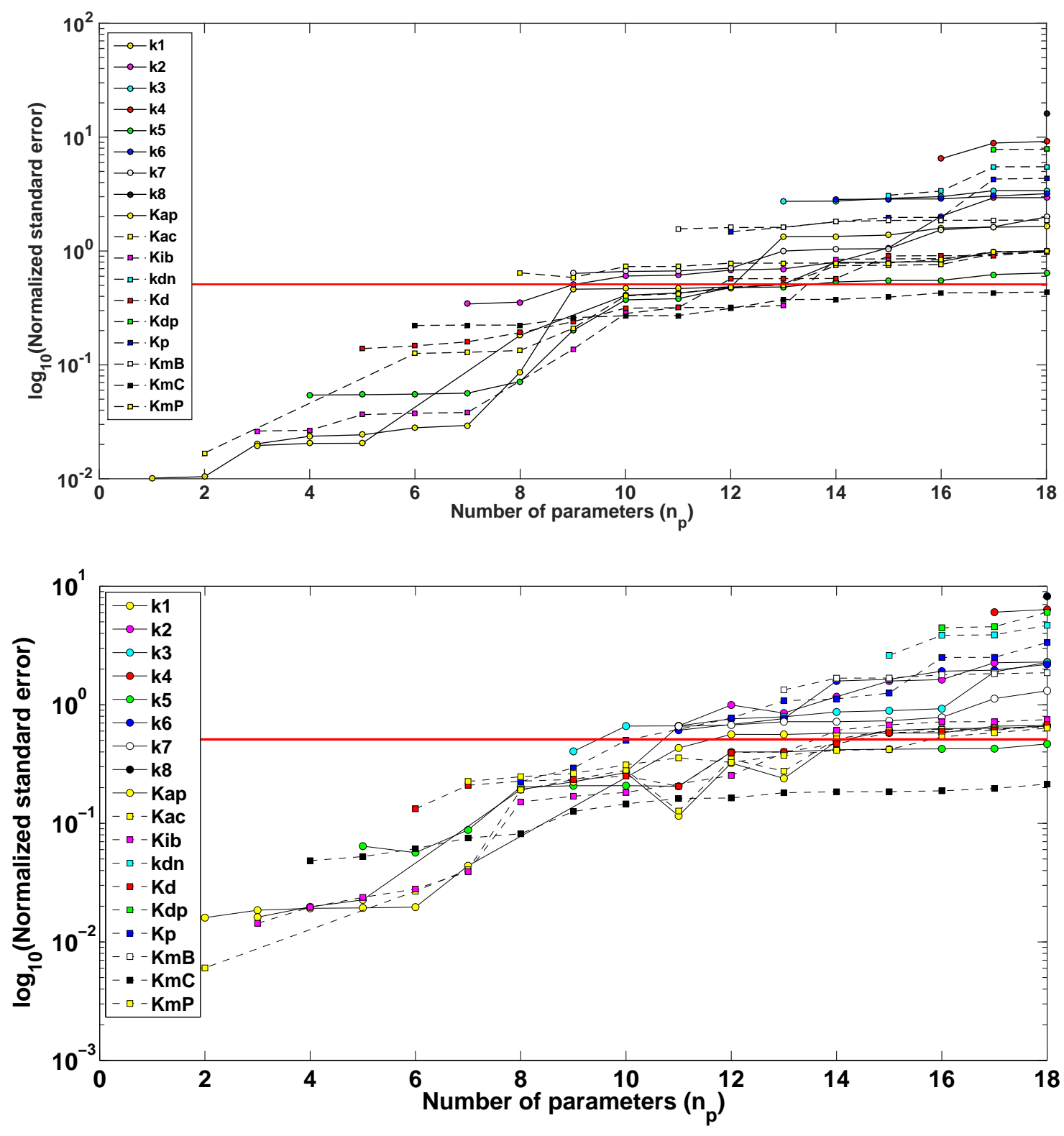

Figure 2: The normalized standard errors (NSEs) associated with a naive (Top) and optimal (Bottom) experimental design using $t_{f}=72 \mathrm{hrs}$, observation frequency $=3 \mathrm{hrs}$, and $H=3$. The solid horizontal line is the threshold for the selection of parameters with reasonable normalized standard errors $(\mathrm{NSE}=0.5102)$. 


\subsection{Optimal Experimental Design: $t_{f}=72 \mathrm{hrs,} \mathrm{observation} \mathrm{frequency}=3 \mathrm{hrs}$, $H=5$}

We investigated whether increasing the number of possible time intervals for the RNAi perturbation could have a greater affect on reducing parameter uncertainty. We implemented our optimization algorithm using an experimental time of 72 hours, an observation frequency of 3 hours, and 5 time intervals for possible RNAi activation/deactivation $(H=5)$. Using 5 possible perturbation intervals, we found that the optimal time mesh $s$ and corresponding perturbation vector $b$ defining $u(t ; b, s)$ were equal to $\{0,16.7561,38.7894,44.5964,70.0176,72\}$ and $\{0,1,1,0,0\}$, respectively. Figure 3 (Top) displays the naive experimental design results with comparison to the optimized experimental design results (Bottom) with regard to parameter uncertainty as calculated using the subset selection algorithm.

We found that, although an optimal experimental design with $H=5$ did have an impact on parameter uncertainty as compared to the naive experimental design, it was not very different from the case with $H=3$ (Figure 3). For example, the sum of the NSEs for $H=3$ and $H=5$ for $n_{p}=18$ were 43.2897 and 43.1881, respectively. Likewise, the maximum number of parameters that lay below the uncertainty threshold for an optimized experimental design was the same for $H=3$ and $H=5$, i.e., $n_{p}=10$.

Instead of affecting parameter uncertainty, we found that increasing $H$ from 3 to 5 changes the timing of the optimal RNAi perturbation, from to 16.84-47.08 hours to 16.75-44.59 hours (see Figure 4 for forward solutions). Interestingly, the RNAi perturbation was not equivalent for the $H=3$ and $H=5$ case, despite the observation that both experimental designs resulted in essentially a single continuous perturbation. This result may reflect the non-uniqueness of the solution to the optimization algorithm, which, in general, is not guaranteed by optimal experimental design theory. Thus, we surmise that the optimal solution to the design problem is highly dependent on the free parameters used to implement the optimization algorithm, e.g., the choice of $H$. 

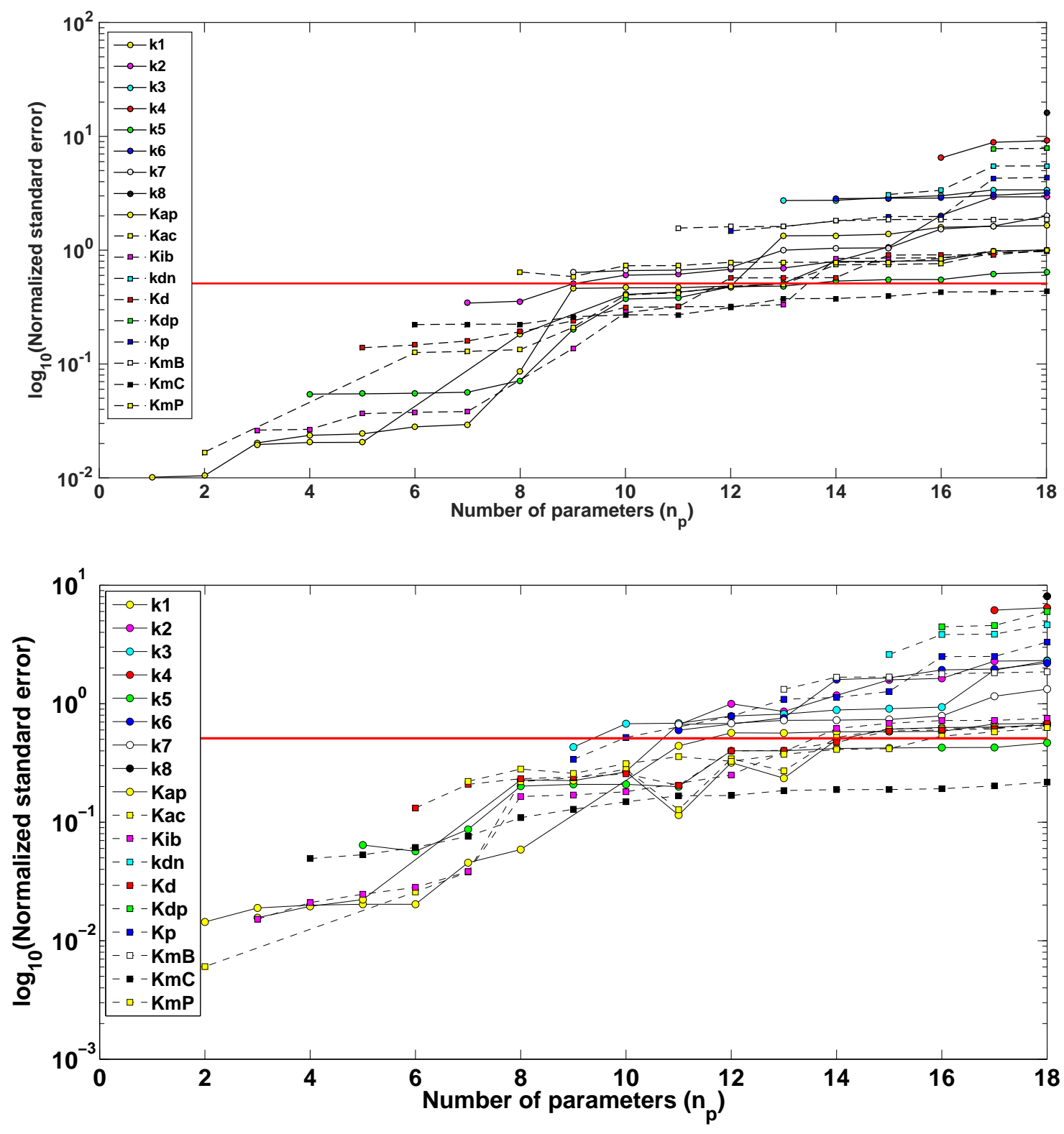

Figure 3: The normalized standard errors (NSEs) associated with a naive (Top) and optimal (Bottom) experimental design using $t_{f}=72 \mathrm{hrs}$, observation frequency $=3 \mathrm{hrs}$, and $H=5$. The solid horizontal line is the threshold for the selection of parameters with reasonable normalized standard errors $(\mathrm{NSE}=0.5102)$. 

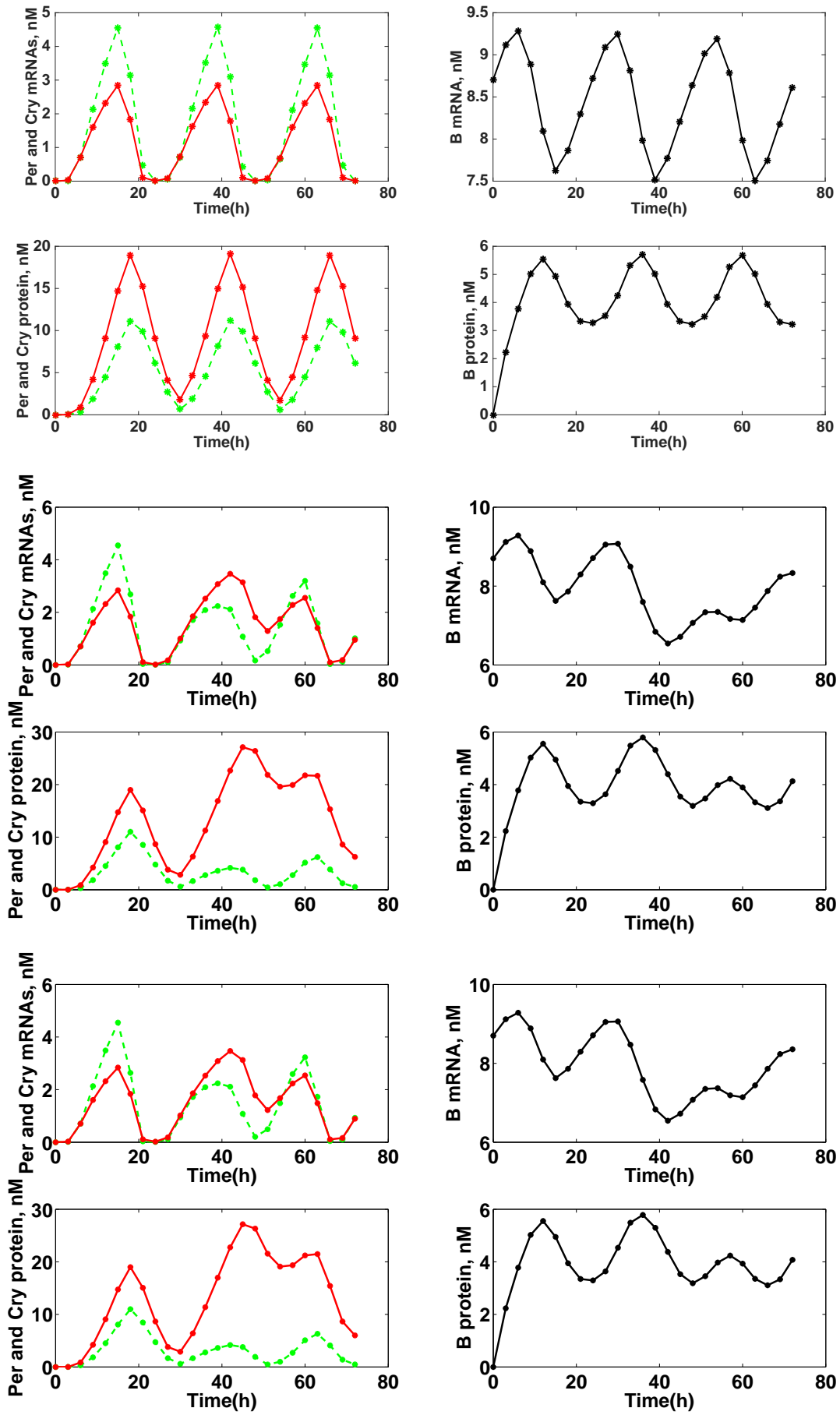

Figure 4: Forward solutions of the circadian model for the naive and optimized experimental designs for the Per, Cry, and Bmal1 (B) mRNAs and PER, CRY, and BMAL1 (B) proteins from 0-72 hours with the observation frequency equal to 3 hours. In the left figures, solid lines represent Cry while dashed lines are Per. Forward solutions are calculated at discrete observation time points (every 3 hours) and plotted as $\left(^{*}\right)$, with connecting lines shown for clarity. Top (4 plots): Forward solutions for the naive experimental design. Middle (4 plots): Forward solutions for the optimized experimental design $(H=3)$. Bottom (4 plots): Forward solutions for the optimized experimental design $(H=5)$. 


\subsection{Optimal Experimental Design: $t_{f}=72 \mathrm{hrs}$, observation frequency $=1 \mathrm{hrs,}$ $H=3$}

We investigated whether increasing the observation frequency from every 3 hours to every hour could have a greater affect on reducing parameter uncertainty when using our optimal design algorithm with $H=3$. Using 3 possible perturbation intervals with a one hour observation frequency, we found that the optimal time mesh $s$ and corresponding perturbation vector $b$ defining $u(t ; b, s)$ were equal to $\{0,15.6869,44.0767,72\}$ and $u(t)=\{0,1,0\}$, respectively. Figure 5 (Top) displays the naive experimental design results with comparison to the optimized experimental design results (Bottom) with regard to parameter uncertainty as calculated using the subset selection algorithm.

Our results indicate that increasing the observation frequency when utilizing our optimal design algorithm may have a significant affect on lowering parameter uncertainty. In the case of $H=3$, the sum of the NSEs for $n_{p}=18$ parameters was lowered from 43.28 to 25.41 by increasing the observation frequency from 3 per hour to 1 per hour, respectively. The maximum number of parameters that lay below the uncertainty threshold for an optimized experimental design with 3 and 1 hour observation frequencies was $n_{p}=10$ and $n_{p}=12$, respectively. There are 10 parameters below the uncertainty threshold at $n_{p}=18$ when using an optimized experimental design with 1 hour observation frequency and $H=3$ (Figure 5 (Bottom)). In contrast, there are only 2 parameters that lie below the uncertainty threshold at $n_{p}=18$ when using an optimized experimental design with 3 hour observation frequency and $H=3$ (Figure 2 (Bottom)).

We note that increasing the observation frequency when using a naive experimental design could have a similar affect on parameter uncertainty as using an optimal experimental design. For example, we found that increasing the observation frequency to every hour when using a naive experimental design resulted in parameter uncertainty levels similar to results found when using an optimal experimental design with a 3 hour observation frequency and $H=3$. For example, there are two parameters that lie below the uncertainty threshold at $n_{p}=18$ for both of these cases (compare Figure 5 (Top) and Figure 2 (Bottom)).

We also tested whether increasing the number of perturbation time intervals from $H=3$ to $H=5$ could have an affect on parameter uncertainty when using a 1 hour observation frequency. Similar to the case with a 3 hour observation frequency, we found that increasing $H$ had only a minor influence on overall parameter uncertainty. Specifically, the sum of NSEs for $n_{p}=18$ was equal to 25.1412 when $H=3$ and slightly decreased to 25.1411 when $H=5$ (parameter subset selection plots not shown). 

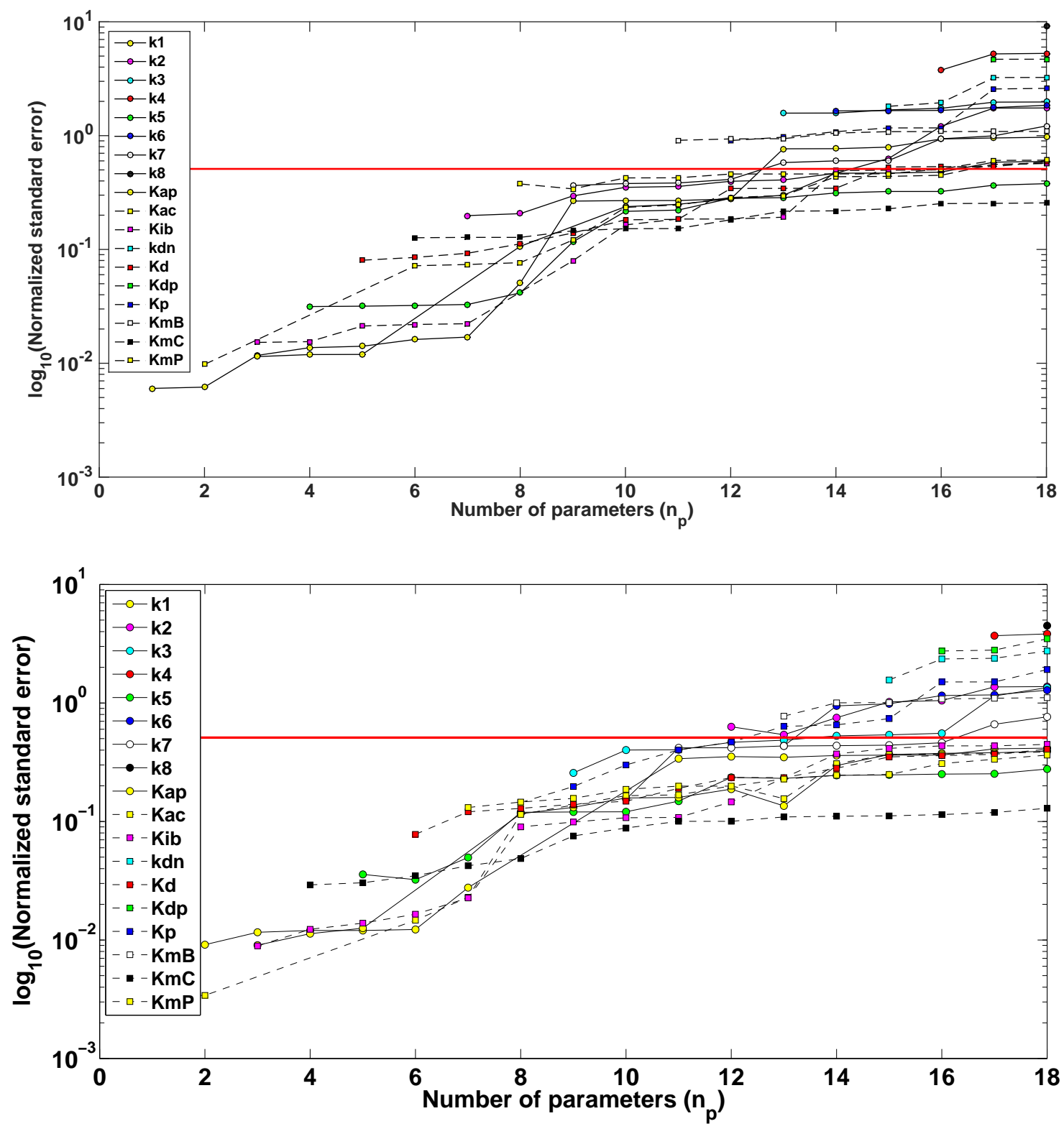

Figure 5: The normalized standard errors (NSEs) associated with a naive (Top) and optimal (Bottom) experimental design using $t_{f}=72 \mathrm{hrs}$, observation frequency $=1 \mathrm{hr}$, and $H=3$. The solid horizontal line is the threshold for the selection of parameters with reasonable normalized standard errors $(\mathrm{NSE}=0.5102)$. 


\subsection{Optimal Experimental Design: $t_{f}=320 \mathrm{hrs}$, observation frequency $=3 \mathrm{hrs,}$ $H=3$}

We investigated whether increasing the total experiment time from $t_{f}=72$ hours to $t_{f}=320$ hours could have an affect on reducing parameter uncertainty when using our optimal design algorithm with $H=3$. Using 3 possible perturbation intervals with a 3 hour observation frequency, we found that the optimal time mesh $s$ and corresponding perturbation vector $b$ defining $u(t ; b, s)$ were equal to $\{0.0005,108.7691,210.8853,313.3115\}$ and $\{0,1,0\}$, respectively. Figure 6 (Top) displays the naive experimental design results with comparison to the optimized experimental design results (Bottom) with regard to parameter uncertainty as calculated using the subset selection algorithm.

Our results indicate that increasing the total experiment time when utilizing our optimal design algorithm may have a significant affect on lowering parameter uncertainty. For example, in the case of $H=3$, the sum of the NSEs for $n_{p}=18$ parameters was lowered from 43.28 to 11.3029 by increasing $t_{f}$ from 72 to 320 , respectively (compare Figure 2 (Bottom) to Figure 6 (Bottom)). The maximum number of parameters that lay below the uncertainty threshold for an optimized experimental design for $t_{f}=72$ and $t_{f}=320$ hours were $n_{p}=10$ and $n_{p}=14$, respectively. Likewise, the number of parameters that were below the uncertainty threshold at $n_{p}=18$ were 2 and 11 for the case when $t_{f}=72$ and $t_{f}=320$, respectively.

We plotted the forward solutions of the circadian system with a naive or optimized experimental design and total experiment time of 320 hours (Figure 7). Examining these plots, and comparing to Figure 4, we observed that one of the primary differences between a 72 hour and 320 hour experiment was that a 320 hour experiment allows the circadian system more time to return to its periodic equilibrium. These results suggest that a longer experiment time may provide more information content to a data set, not only by increasing the number of total data points collected, but also by allowing the experimenter to more fully observe different dynamic regimes when employing system perturbations. 

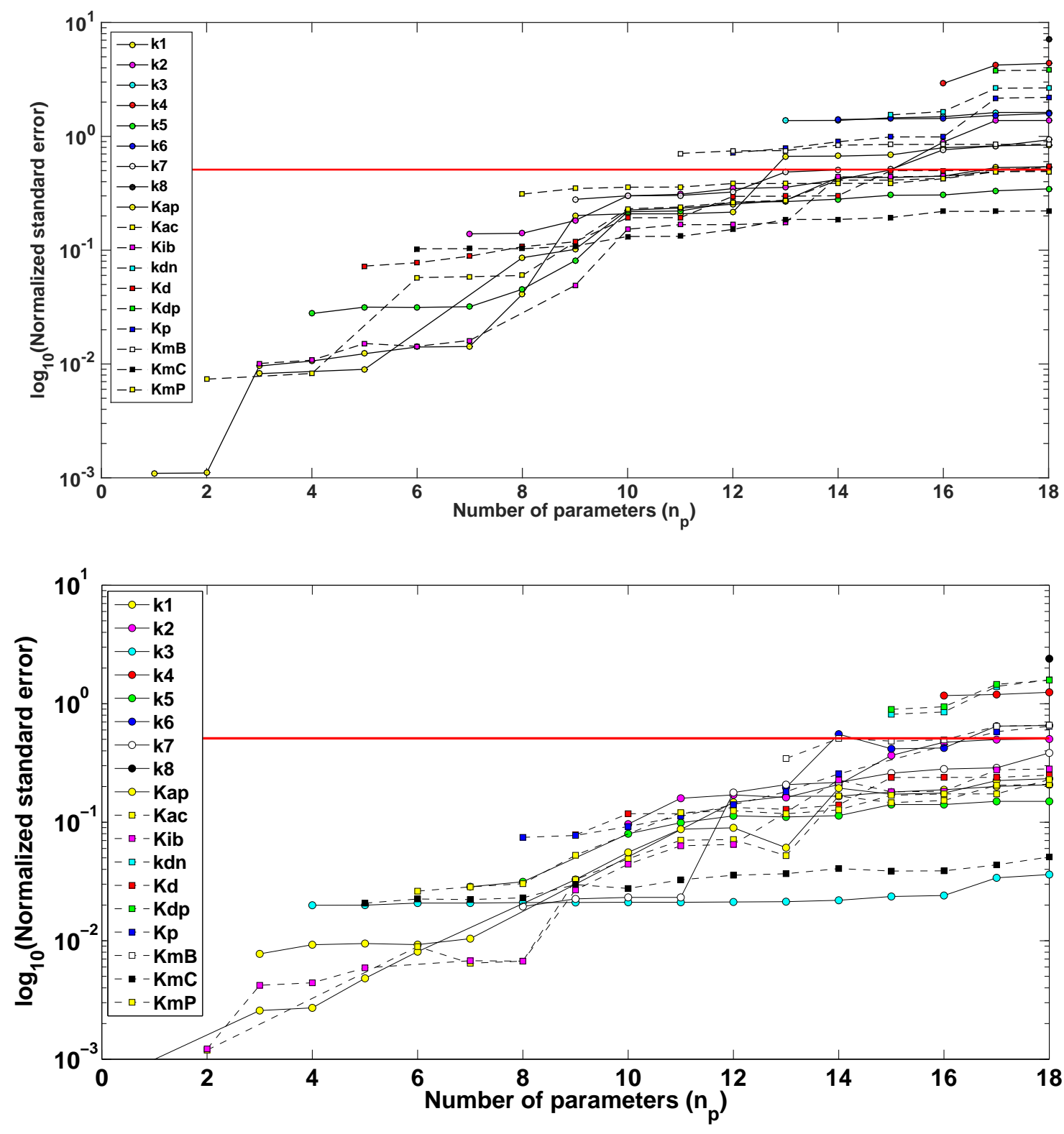

Figure 6: The normalized standard errors (NSEs) associated with a naive (Top) and optimal (Bottom) experimental design using $t_{f}=320 \mathrm{hrs}$, observation frequency $=3 \mathrm{hrs}$, and $H=3$. The solid horizontal line is the threshold for the selection of parameters with reasonable normalized standard errors $(\mathrm{NSE}=0.5102)$. 

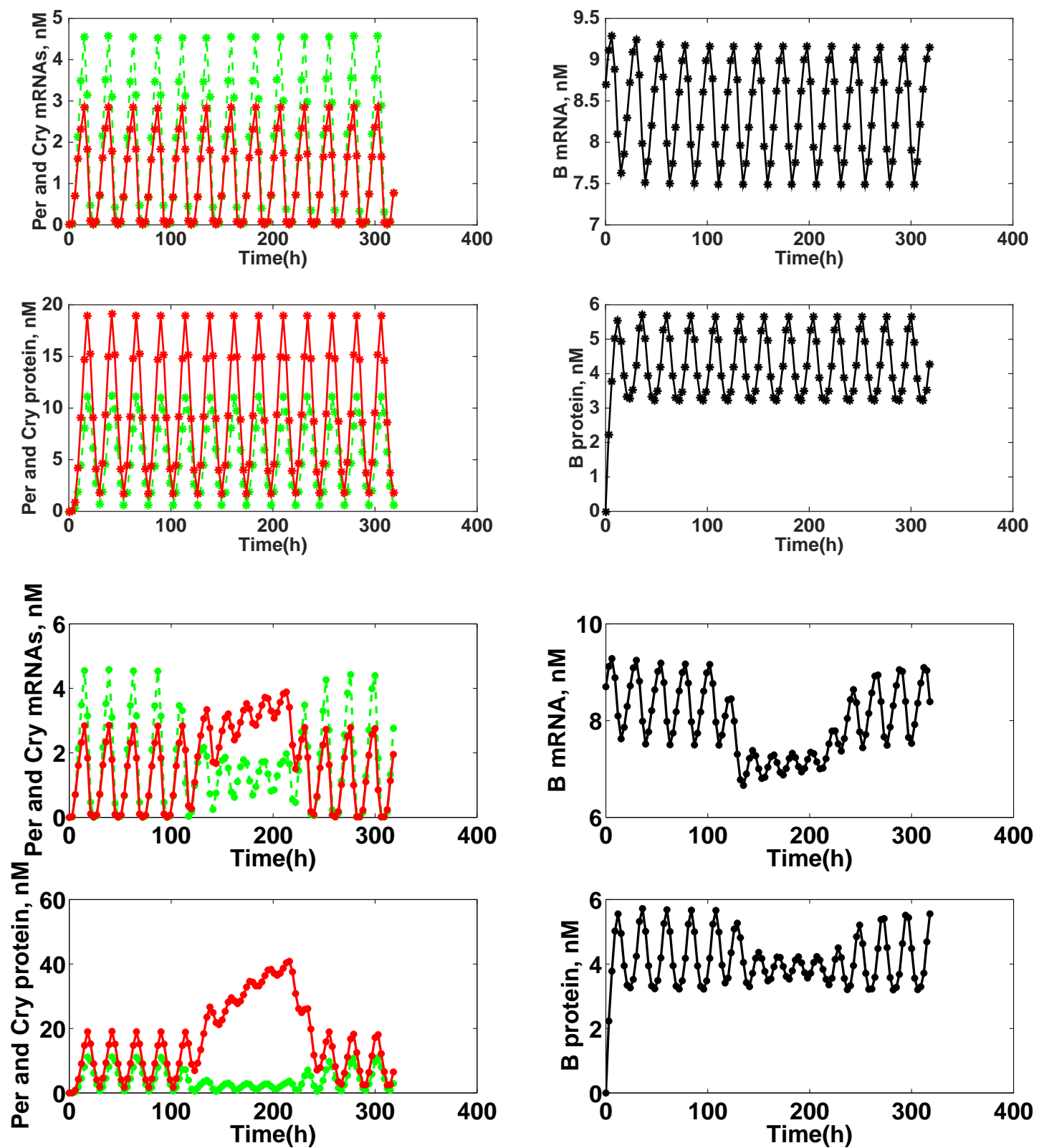

Figure 7: Forward solutions of the circadian model for the naive and optimized experimental designs for the Per, Cry, and Bmal1 (B) mRNAs and PER, CRY, and BMAL1 (B) proteins from 0-320 hours with the observation frequency equal to 3 hours. In the left figures, solid lines represent Cry while dashed lines are Per. Forward solutions are calculated at discrete observation time points (every 3 hours) and plotted as $\left(^{*}\right)$, with connecting lines shown for clarity. Top (4 plots): Forward solutions for the naive experimental design. Bottom (4 plots): Forward solutions for the optimized experimental design $(H=3)$. 


\section{Discussion}

Overall, our results suggest that experimental input manipulation that produces non-equilibrium system dynamics for oscillatory systems yields more informative data sets for parameter estimation than that obtained when using an unaltered or undisturbed system. In particular we obtain more statistical certainty in parameters for the model in the form of lower normalized standard errors. Optimization of the input manipulation with a piecewise constant functional form, i.e., via our iterative algorithm (Equations (29) and (30)), can successfully reduce standard errors in parameter estimates for the circadian system, and possibly among other models with dynamic equilibria. As shown unanimously in the graphs presented in the Results section, creating a perturbation in the dynamic circadian system at least results in lower normalized standard errors and often increases the number of parameters below the designated threshold. Increasing the observation frequency while calculating optimal system perturbations further reduces the normalized standard errors, as seen in Figure 5.

Interestingly, when the duration of the experiment was increased, the normalized standard errors decreased without the perturbation and even more so with the system disturbance. This was evidenced by Figure 6 . The result matches intuition since the observation frequency of 3 hours is not related to the periodicity of the system's oscillations; therefore, when the model is simulated for 320 hours, there is not yet any information repetition or selection of points with the same values at different times. The opposite of this would be selecting the maximum of each period at different times. The data would essentially be observed as constant, and the Fisher Information Matrix would not be invertible. Since the sensitivities are calculated at time points not dependent on the periodicity of the model oscillations, running the model for a longer period of time "discovers" more about the solution, thereby producing a Fisher Information Matrix without any similar columns or rows. As seen in Figure 6, perturbing the system away from this equilibrium only exacerbates this effect.

Future investigations will attempt to investigate possible parameter correlations by bootstrapping and calculating standard errors through asymptotic techniques in the context of information theory. Provided raw data, another future investigation could consolidate the conclusion of this paper with inverse problem techniques and extrapolate these findings to the creation of optimal experimental designs.

\section{Acknowledgements}

This research was supported in part by the Air Force Office of Scientific Research under grant number AFOSR FA9550-12-1-0188, in part by the National Science Foundation by Undergraduate Biomathematics grant number DBI-1129214 and in part by the National Science Foundation under grant number DMS-0946431.

\section{References}

[1] K. Adoteye, H. T. Banks, K. B. Flores, Optimal design of non-equilibrium experiments for genetic network interrogation. CRSC-TR14-12, Center for Research in Scientific Computation, North Carolina State University, September, 2014; Applied Math Letters, 40 (2015), 84-89.

[2] H.T. Banks, Robert Baraldi, Karissa Cross, Kevin Flores, Chistina McChesney, Laura Poag, and Emma Thorpe, Uncertainty quantification in modeling HIV viral mechanics. CRSC-TR13-16, Center for Research in Scientific Computation, North Carolina State University, December, 2013; Mathematical Biosciences and Engineering, to appear.

[3] H. T. Banks, A. Cintron-Arias, and F. Kappel, Parameter selection methods in inverse problem formulation, in Mathematical Modeling and Validation in Physiology, ser. Lecture Notes in Mathematics (eds. J. J. Batzel, M. Bachar, and F. Kappel), Springer Berlin Heidelberg (2013), 43-73.

[4] H. T. Banks, K. B. Flores, K. Link, L. Poag, T. Huffman, and J. Nardini, A mathematical model of RNA3 recruitment in the Brome Mosaic Virus replication cycle, International Journal of Pure and Applied Mathematics, 89(2), (2013) 251-274. 
[5] H. T. Banks, K. Holm, and F. Kappel, Comparison of optimal design methods in inverse problems, Inverse Problems, 27 (2011) 075002.

[6] H. T. Banks, S. Hu, and W. C. Thompson, Modeling and Inverse Problems in the Presence of Uncertainty, CRC Press, Boca Raton (2014).

[7] H.T. Banks and H.T. Tran, Mathematical and Experimental Modeling of Physical and Biological Processes, CRC Press, Boca Raton London New York, 2009.

[8] A. Cintron-Arias, H. T. Banks, A. Capaldi and A. L. Lloyd, A sensitivity matrix based methodology for inverse problem formulation, CRSC Tech. Rep. CRSC-TR09-09, April, 2009; J. Inverse and Ill-posed Problems, 17 (2009), 545-564.

[9] Jean-Christophe Leloup and Albert Goldbeter, Modeling the mammalian circadian clock: Sensitivity analysis and multiplicity of oscillatory mechanisms, Journal of Theoretical Biology, 230 (2004), 541-562.

[10] Joaquim R. R. A. Martins, Ilan M. Kroo, and Juan J. Alonso, An automated method for sensitivity analysis using complex variables, Proc. 38th Aerospace Sciences Meeting, paper AIAA-2000-0689, Amer. Inst. Aeronautics and Astronautics (2000), pp. 1-12.

[11] Edward Yang, et al., Decay rates of human mRNAs: correlation with functional characteristics and sequence attributes, Genome Research, 13 (2003), 1863-1872. 